\title{
Urgent pediatric consultation for otologic conditions
}

\author{
N. Sapundzhiev, I. Zenev, L. Dzhenkova, \\ Department of Otolaryngology, Head and Neck Surgery, \\ Queen Jiovanna University hospital - Sofia, Bulgaria
}

\section{Abstract}

Introduction: Pediatric otologic disorders are one of the most frequent reasons for urgent pediatric consultation, thus demanding important medical resources.

Objective: The aim of the present study was to give quantitative description of the overall otologic pediatric pathology, presenting to an emergency ENT consultations office in the city of Sofia, Bulgaria.

Patients and method: The records of all pediatric patients presented with urgent otorhinolaryngologic symptoms during one calendar year were retrospectively reviewed.

Results: Consultations for otologic conditions in children account for $23.7 \%$ of all cases and for $55.3 \%$ of all pediatric consultations. The leading symptoms were from the external in $14.5 \%$ of the cases, form the middle ear - in $85.2 \%$ and form the inner ear in $0.3 \%$. The most frequent etiologies include trauma for the auricle and infections for the external auditory canal and the middle ear. During the different year seasons there is significant variation in the relative incidence of otitis media and otitis externa. Much larger variations show the incidences of different pathologic otologic conditions in the different age groups. Otologic symptoms present an average of $52.8 \pm 9.4 \%$ of all emergency ENT consultations with an age peak in the age group 3-12 years, reaching up to $66 \%$, followed by a gradual decline.

Conclusion: The observed high proportion of children with otologic symptoms seen at an emergency ENT office underlines the importance of this pathology. The data reported could be used as reference when planing the facilities needed to provide adequate specialised medical help in urban areas.

\section{Introduction}

Diseases of the ear and otologic disorders appear to be one of the most frequent reasons for pediatric consultation $[2,3,10]$. On the other hand they have important economic weight $[4,6]$. The leading symptom is pain, which presents an important psychological burden to the child and the parents, urging them to seek specialised medical advice often outside the regular working hours of the ENT offices [12].

\section{Objective}

On this background the aim of the present study was to evaluate the quantitative characteristics of emergency pediatric ENT consultations in a large urban area in Bulgaria.

\section{Study design}

Retrospective review.

\section{Setting}

The setting for this study was the emergency medical centre at the Queen Jiovanna hospital in Sofia. It covers the city and some small towns nearby. This is the only 24-hour ENT ambulatory office and clinic, which should meet the demands of slightly above 1000000 citizens. This study was undertaken to review our experience with pediatric otology patients. 


\section{Patients and method}

The records of all pediatric patients presented with urgent otorhinolaryngologic symptoms during one calendar year (1st January 2004 till 31st December 2004) were retrospectively reviewed. Details of sex, age, clinical history, findings and course were collected and analysed for all patients. The leading diagnosis in otologic disorders was based on pneumatic otoscopy or ear microscopy.

\section{Results}

Of the total 6341 consultations performed in the emergency otolaryngologic office during the calendar year 2720 or $42.9 \%$ were for children. Of them $1578(58 \%)$ were boys and $1142(42 \%)$ girls with a mean age of $6.3 \pm 4.8$ years (Table 1 ). The sex difference observed corresponds to other reports on pediatric otitis media [13]. However this inequality is still debatable and has not been given etiologic explanation $[3,5]$. In the majority of cases the reason for consultation were otologic symptoms $(55.3 \%)$. Of the 1503 children presenting with otologic symptoms in $218(14.5 \%)$ the cause was involvement of the external ear, in 1281 $(85.2 \%)$ of the middle year and in $4(0.3 \%)$ - of the inner ear (Fig. 1).

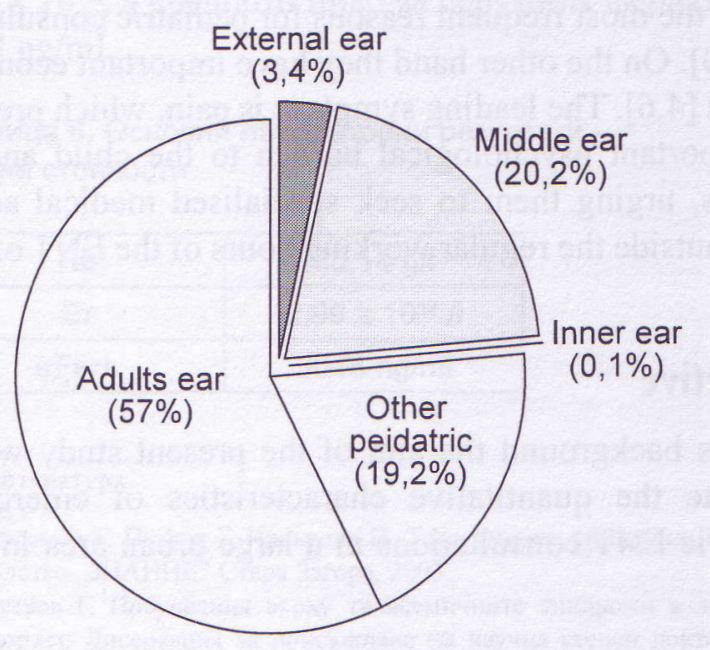

Figure 1: General profile of all patients seen at the emergency ENT office over one year. Otologic conditions in children account for a total of $23.7 \%$ of all cases.

Of the children presenting with pathologic conditions of the external ear in $25(11.5 \%)$ the auricle was involved. The leading etiologies included traumatic injuries (68\%), in the rest cases - infectious conditions of the skin and deeper tissues (1 $\mathrm{x}$ erysipel, $3 \mathrm{x}$ atheroma, $1 \mathrm{x}$ eczema and $3 \mathrm{x}$ perichondritis). In the rest $88.5 \%$ of the cases with leading involvement of the external ear the primary site was the external auditory canal (EAC). Here the infectious etiology was leading accounting for $81 / 192$ cases $(42 \%)$ and included 77 cases of otitis externa and 4 cases of mycosis. The traumatic cases were only $22 / 192(11.4 \%)$. Impressing is the important incidence of foreign bodies in the EAC - 56/192 (29.2\%). In further 34 cases the otologic examination revealed nothing particular apart of obstruction of the EAC by cerumen.

The largest group of otologic disorders in children, which led to emergency consultation, includes diseases of the middle ear. Even on the scale of all ENT consultations for any cause and any age they represent an important proportion $-20.2 \%$ (Fig. 1). Acute otitis media formed an overwhelming proportion of the cases, accounting for $95 \%(1216 / 1281)$ of the cases. The reminder was formed by otitis media with effusion (29/1281), traumatic perforations (25/1281), chronic OM (8/1281) and unclear otalgia (3/1281) (Fig. 2). Acute otitis media presented under several different forms. In $83.5 \%$ the findings corresponded to acute otitis without effusion - mild inflammation of the tympanic membrane with vessel dilation and redness but without evident signs of liquid collection behind the eardrum. However the range of the findings in this clinical entity was quite large [1]. The second important group presented the acute otitis media with clear evidence of effusion (most often purulent) under pressure in the ear cavity $(166 / 1216)$ $13.7 \%$. Two of these cases had acute mastoiditis too. In $35 / 1218(2.9 \%)$ the acute otitis presented as myringitis bullosa. The exact etiology of this condition is nowadays still debatable, but undoubtfully it belongs to the group of infectious conditions of the ear $[8,14]$. Apart of the 25 cases of traumatic perforation of the ear drum all other pathologic conditions of the middle ear seen at the emergency ENT consultant office were of infectious etiology $1256 / 1281$ (98\%).

During the year there were only 4 cases of pediatric consultation because of pathologic conditions with primary involvement of the internal ear -3 cases with sudden hearing loss and 1 case of tinnitus.

Otologic pathology accounts for different proportion of the total pediatric cases throughout the year. The percentage varies form $47.7 \%$ to $69.8 \%$ (range 22) with two peaks (in spring and late autumn) and two drops (beginning of the year and late summer) (Fig. 3). The most important variations show the relative incidence of otitis media and otitis externa. The average incidence of otitis media ranges between $61.4 \%$ and $90.7 \%$ with a single clear minimum in late summer, on the background of relatively constant level during the rest of the year $-82.9 \pm 10.1 \%$. These variations mirror those of otitis externa. Here the average level is constantly low $-16.8 \pm 9.8 \%$ with a 


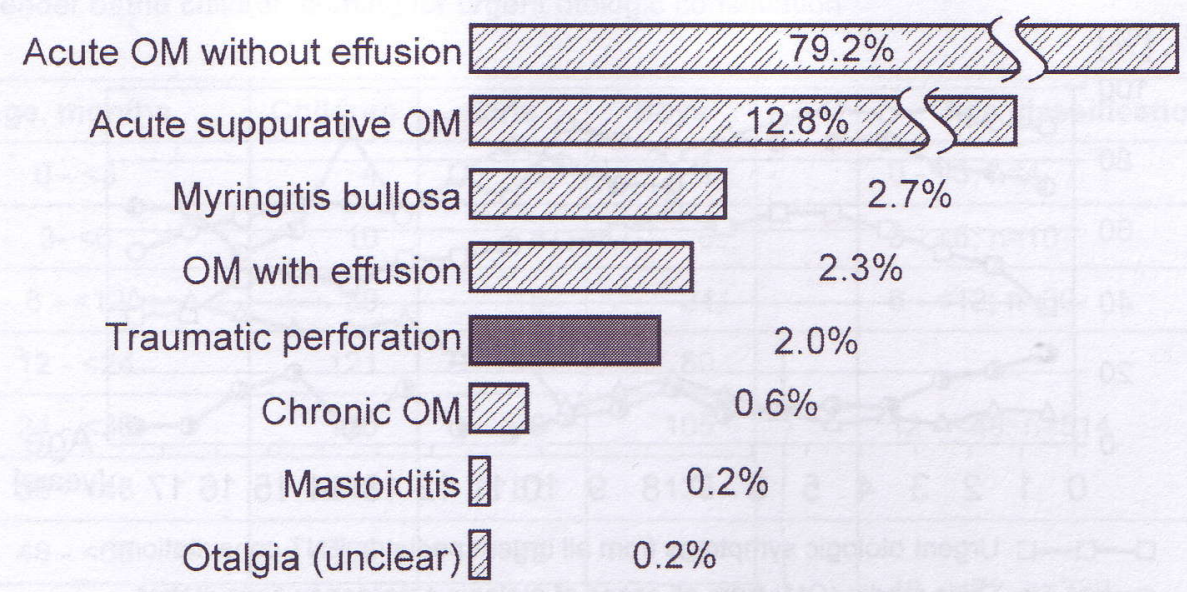

Figure 2: Relative incidence of the different pathologic conditions of the middle ear in the pediatric population, which led to urgent ENT consultation.

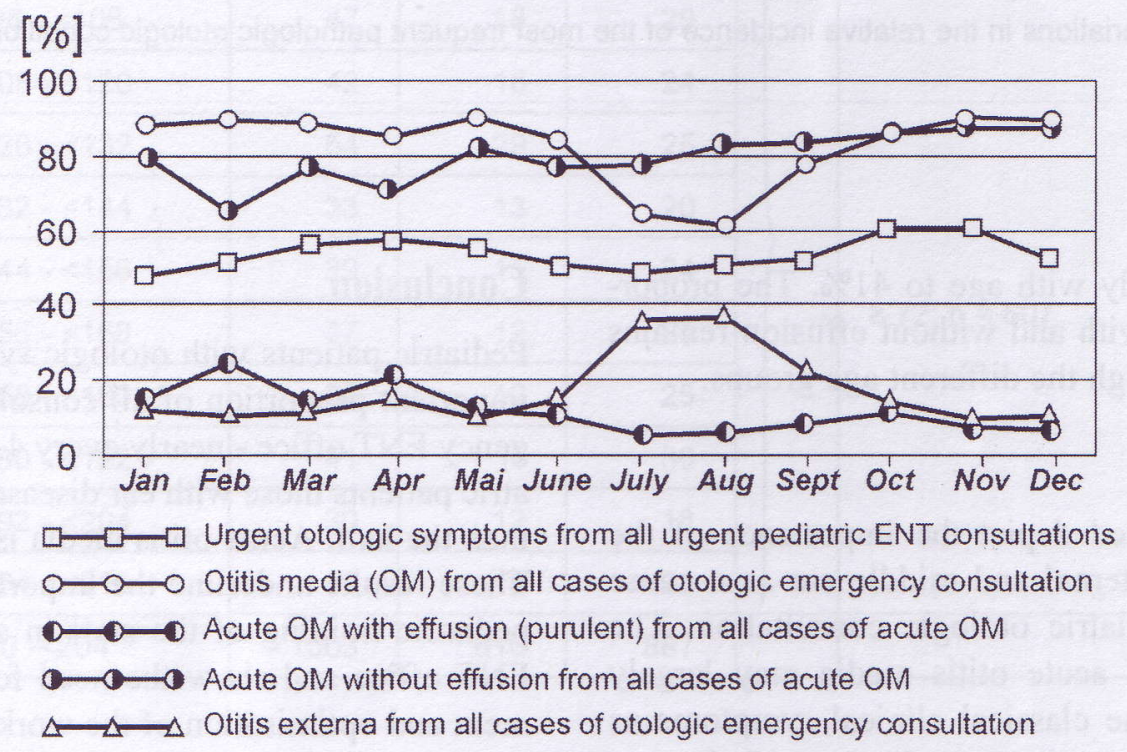

Figure 3: Seasonal variations in the relative incidence of the most frequent pathologic otologic conditions in the pediatric population, which led to urgent ENT consultation.

marked rise in July-August to a level $36.8 \%$ (range 9.3-36.8\%). Joined reciprocal variation show also the relative incidence of acute otitis media with effusion (suppurative) and without effusion calculated as a part of all cases of acute otitis media. The relative incidence of the purulent form is higher at the end of the winter season $(24.5 \%$ ), dropping as low as $5.6 \%$ in the summer, whereas the relative incidence of $\mathrm{OM}$ catarrhalis is lower in winter $(64.7 \%)$ with an increase in summer/autumn to $87.6 \%$.

Much larger variations show the incidences of different pathologic otologic conditions in the different age groups. Otologic symptoms present an average of $52.8 \pm 9.4 \%$ of all emergency ENT consultations (Fig. 4). The age peak is in the age interval 3-12 years, reaching up to $66 \%$, followed by a gradual decline. The results of other sources reveal that similar increase with age is actually a cumulative value of children with recurrent AOM. The peak of the first episode of AOM is at the age of 1-2 years [3-5]. The design of our study was however insensitive to detect and measure the impact of repeat consultation for the total age incidence. A similar trend of increase with age is observed for the most common etiology - infectious conditions. Again the most important variations show the relative incidence of otitis media and otitis externa. The incidence of otitis media is quite high in the early children age (94.3\%) and constantly slowly decreasing in elder children to drop to only $53.8 \%$ till the age of 18 . The trend in otitis externa is reciprocal. Inflammation of the external ear is quite rare in babies and small children $(5.7 \%)$ and increases its relative 


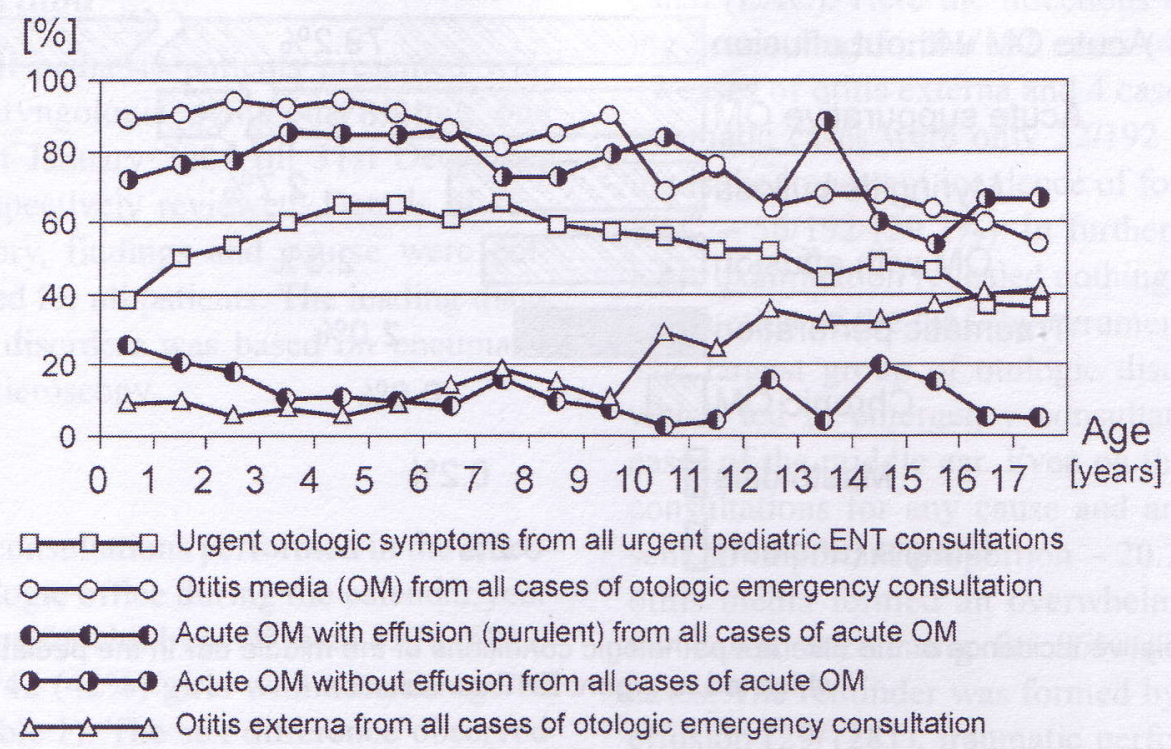

Figure 4: Variations in the relative incidence of the most frequent pathologic otologic conditions with age.

importance gradually with age to $41 \%$. The proportion of acute OM with and without effusion remains quite constant through the different age groups.

\section{Discussion}

The results presented depict the importance of the disorders of the external and middle ear as a cause for emergency pediatric otologic consultation. The clinical findings in acute otitis media may largely vary. In children the classical clinical symptoms as pain, rubbing of the ear etc. are often specific but too insensitive while he general symptoms as rhinitis, irritability, fever, poor appetite etc. are both insensitive and unspecific [1]. Young infants are however unable to express the local or general symptoms and the judgement is based solely on the otoscopic findings $[1,4]$. This could explain the frequent referral of children by general practitioners and pediatricians to ENT specialists in which the otologic findings are normal. Sometimes it could be a hard task to convince the parents, that their child does not suffer ear disease and they should return to the GP for further investigations/tests $[4,11]$. The incidence of acute mastoiditis appeared very low with only 2 cases in 1503. Similar incidence is reported by Petersen et al. in a Danish population [9]. Our observations corresponds to the general trend of declining incidence of mastoiditis observed during recent decades

\section{Conclusion}

Pediatric patients with otologic symptoms present an important proportion of all consultations in an emergency ENT office - nearly every 4-th one. Of all pediatric patients those with ear diseases account for more than the half. Acute otitis media is the leading cause. These results underline the importance of the special pediatric training of the staff in such an emergency ENT office and show the need for particular equipment and optimisation of the workflow, which is also in accordance with analysis reports from countries with other health system $[7,10]$.

The incidence of the different ear diseases varies to some extent depending on the year season. Much stronger are the age trends. Further studies should compare the observed incidences and trends with those from normal ENT offices as well from other urban centres from Bulgaria to help plan the facilities needed to provide adequate specialised medical help. 
Table 1: Age and gender of the children coming for urgent otologic consultation

\begin{tabular}{|c|c|c|c|c|}
\hline Age, months & Children & Girls & Boys & Age classification \\
\hline $0-<3$ & 4 & 3 & 1 & $0-<3 ; n=4$ \\
\hline $3-<6$ & 10 & 4 & 6 & $3-<6 ; n=10$ \\
\hline $6-<12$ & 39 & 15 & 24 & $6-<12 ; n=39$ \\
\hline $12-<24$ & 121 & 41 & 80 & \multirow{3}{*}{$12-<48 ; n=514$} \\
\hline $24-<36$ & 175 & 70 & 105 & \\
\hline $36-<48$ & 218 & 82 & 136 & \\
\hline $48-<60$ & 186 & 79 & 107 & \multirow{2}{*}{$48-<72 ; n=329$} \\
\hline $60-<72$ & 143 & 68 & 75 & \\
\hline $72-<84$ & 109 & 52 & 57 & \multirow{12}{*}{$\geq 72 ; n=607$} \\
\hline $84-<96$ & 99 & 44 & 55 & \\
\hline $96-<108$ & 47 & 18 & 29 & \\
\hline $108-<120$ & 42 & 18 & 24 & \\
\hline $120-<132$ & 54 & 29 & 25 & \\
\hline $132-<144$ & 33 & 13 & 20 & \\
\hline $144-<156$ & 39 & 15 & 24 & \\
\hline $156-<168$ & 37 & 12 & 25 & \\
\hline $168-<180$ & 37 & 12 & 25 & \\
\hline $180-<192$ & 41 & 11 & 30 & \\
\hline $192-<204$ & 30 & 12 & 18 & \\
\hline $204-<216$ & 39 & 18 & 21 & \\
\hline $0-<204$ & 1503 & 616 & 887 & \\
\hline
\end{tabular}

\section{References:}

1. Alho O, Koivunen P, Luotonen J. Diagnostic criteria for otitis media in children. Otorhinolaryngol Nova 1998; 8: 123-128.

2. Charles J, Pan Y, Britt H. Trends in childhood illness and treatment in Australian general practice, 1971-2001. Med J Aust 2004; 180: 216-9.

3. Garces-Sanchez M, Diez-Domingo J, Alvarez de Labiada T, Planelles V, Graullera M, Baldo JM, Garcia Llop LA, Garcia Lopez M, Peris Vidal A, Gallego Garcia MD, Ballester Sanz A, Peidro C, Villarroya J, Jubert A, Colomer Revuelta J, Casani C. Epidemiologia e impacto de la otitis media aguda en la Cominidad Valenciana. An Pediatr (Barc) 2004; 60: 125-32.

4. Grenier B. Otitis media in children: a primer for clinical decision making. Otorhinolaryngol Nova 1998; 8: 129-135.

5. Homoe P, Christensen R, Bretlau P. Acute otitis media and age at onset among children in Greenland. Acta Otolaryngol 1999; 119: 65-71.

6. Howard D, McGowan J. Initial and follow-up costs by treatment outcome for children with respiratory infections. Pediatrics 2004; 113 1352-1356.

7. Hussain $\mathrm{M}$, White $\mathrm{P}$. Consultant led otolaryngology emergency service. ENT News 2004; 13: 63-64.
8. Marais J, Dale B. Bullous myringitis: a review. Clin Otolaryngol 1997; 22: 497-499.

9. Petersen C, Ovesen T, Pedersen C. Acute mastoidectomy in a Danish county from 1977 to 1997 - operative findings and long-term results. Acta Otolaryngol 2000; 122-126

10. Pichichero M. Diagnostic acuuracy, tympanocentesis training performance, and antibioti selection by pediatric residents in management of otitis media. Pediatrics 2002; 110: 1064-1070.

11. Raghavan U, NS. J. Combating bacterial resistance in otorhinolaryngology. Clin Otolaryngol 2002; 27: 446-452.

12. Sarrell E, Cohen H, Kahan E. Naturopathic treatment for ear pain in children. Pediatrics 2003; 111: e574-e579.

13. Tekin M, Schacherin P, Mutlu C, Jaisinghani J, Paparella M, Le C Purulent otitis media in children and adults. Eur Arch Otorhinolaryngol 2002; 259: 67-72.

14. Wild D, Spraggs P. Myringitis bullosa haemorrhagica associated with meningo-encephalitis. Eur Arch Otorhinolaryngol 2003; 260: 320-321. 